Objective To determine the trend in the syndromic diagnosis of sexually transmitted infections (STI).

Methods Data of patients attending our STD clinic between 2008 and 2010 was retrieved. Aetiological diagnosis of individual patients as practiced in our clinic were fit into any of the following syndromic diagnoses-vaginal/cervical discharge, non-herpetic genital ulcer, herpetic ulcer, lower abdominal pain, urethral discharge, ano-rectal discharge, inguinal bubo, scrotal swelling, genital warts, and other STIs that could not be characterised into above syndromes. The syndromic approach as suggested by National AIDS Control Organization of India was followed. Trend of syndromic diagnosis of STIs was determined.

Results In 2008, majority (51.1\%) of the new patients registered to the clinic (36, 26.66\% herpetic genital ulcer, 33, 24.44\% genital warts) had one or the other viral STI. In $2009,43.68 \%$ patients had viral STIs (21.35\% herpetic GUD, 22.33\% genital warts). In 2010, there was a significant increase in the incidence of viral STIs; $72.6 \%$ patients (36.94\% herpetic GUD and $35.66 \%$ genital warts) had one or the other viral syndromic diagnosis.

Conclusion In concurrence to observations worldwide, proportion of viral STIs have increased significantly recently due to widespread use of broad spectrum antibiotics. One more reason could be recurrent or persistent nature of these viral STIs. In our setting, incidences of herpetic GUD and genital warts were almost similar.

\section{P1-S1.24 LONGITUDINAL TRENDS IN SYNDROMIC STI DIAGNOSES IN LILONGWE, MALAWI: 2006-2010}

\section{doi:10.1136/sextrans-2011-050108.24}

${ }^{1} \mathrm{G}$ Kamanga, ${ }^{2} \mathrm{~K}$ Powers, ${ }^{1} \mathrm{C}$ Mapanje, ${ }^{1} \mathrm{~N}$ Mkandawire, ${ }^{1} \mathrm{H}$ Milonde, ${ }^{1} \mathrm{H}$ Kanyamula, ${ }^{1} \mathrm{P}$ Wiyo, ${ }^{1} \mathrm{~F}$ Martinson, ${ }^{2} \mathrm{~W}$ Miller, ${ }^{2} \mathrm{H}$ Hoffman. ${ }^{1}$ UNC Project Malawi, Lilongwe, Malawi; ${ }^{2}$ University of North Carolina, Chapel Hill, USA

Background To understand longitudinal trends in sexually transmitted infection (STI) syndromes, we conducted a descriptive analysis of syndromic STI diagnoses at an STI clinic in Lilongwe, Malawi over the last 5 years.

Methods Using data collected routinely at all STI clinic visits since March 2006, we calculated the proportion of patients seen in each of the last five calendar years (2006, 2007, 2008, 2009, and 2010) who were diagnosed with each of the following STI syndromes-genital ulcer disease, genital warts, abnormal vaginal discharge (females), lower abdominal pain (females), urethral discharge (males), balanitis (males), swollen inguinal lymph nodes (males), and scrotal swelling (males).

Results An average of 6694 unique patients were seen each year; approximately $60 \%$ were female and $40 \%$ were male. Among males, the most common diagnoses were urethral discharge, genital ulcer disease, and balanatis (Abstract P1-S1.24 figure 1). There was an increase in the prevalence of urethral discharge over the period 2006-2010 (from 28\% to $33 \%, p<0.0001$ ), but there was a slight decrease in the prevalence of genital ulcer disease (from 22\% to $20 \%$, $\mathrm{p}=0.02$ ) and balanitis (from $13.5 \%$ to $12 \%, \mathrm{p}=0.0002$ ). Among females, the most common diagnoses were abnormal vaginal discharge, lower abdominal pain, and genital ulcer disease. There was an increase in the prevalence of abnormal vaginal discharge over the period $2006-2010$ (from $40 \%$ to $45 \%$, p <0.0001), but there was a decrease in the prevalence of lower abdominal pain (from $50 \%$ to $33 \%, \mathrm{p}<0.0001$ ) and genital ulcer disease (from 13\% to $10 \%$, $\mathrm{p}<0.0001)$. The prevalence of all other STI syndromes remained below $10 \%$ throughout the study period.

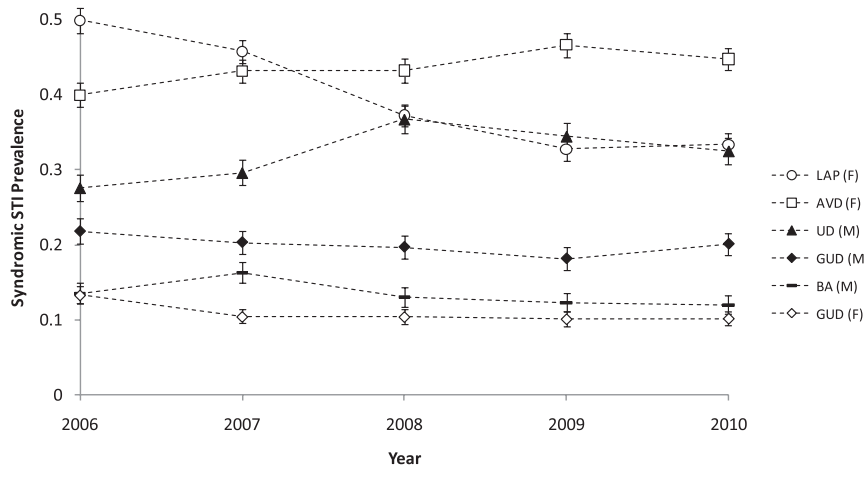

$\angle A P=$ lower abdominal pain,$A V D=$ abnormal vaginal discharge,$U D=$ urethral discharge,$G U D=$ genital ulcer disease, $\mathrm{BA}=$ balanitis, $\mathrm{F}=$ females, $\mathrm{M}=$ males

Abstract P1-S1.24 Figure 1 Syndromic STI prevalence and 95\% Cl, Kamuzu Central Hospital STI Clinic, Lilongwe, Malawi, 2006-2010. LAP, lower abdominal pain; AVD, abnormal vaginal discharge; UD, urethral discharge; GUD, genital ulcer disease; $B A$, balanitis; $F$, female; $M$, male.

Conclusions Urethral discharge and balanitis continue to be highly prevalent among males; abnormal vaginal discharge and lower abdominal pain continue to be highly prevalent among females; and genital ulcer disease continues at a high prevalence in both groups. Of note, the decline in lower abdominal pain coincides with a documented drop in cases of gonorrhoea and chlamydial infection in this population, and the small decline in genital ulcer disease may be due to recent improvements in chancroid treatment. The very high prevalence of urethral discharge among men and abnormal vaginal discharge among women may be due in part to observed high levels of trichomoniasis in this population, and the increases in these diagnoses suggest that trichomoniasis prevalence may be on the rise.

\section{P1-S1.25 TREND OF SEXUALLY TRANSMITTED DISEASES DIAGNOSED BY SYNDROMIC APPROACH IN AN INSTITUTE SETTING}

doi:10.1136/sextrans-2011-050108.25

A Kanwar. Postgraduate Institute of Medical Education and Research, Chandigarh, India

Background The paradigm of research in sexually transmitted diseases (STD) has recently shifted to HIV/AIDS. Recent trend of sexually transmitted diseases in India is lacking.

Objective To determine the trend in the syndromic diagnosis of sexually transmitted infections (STI).

Methods Data of patients attending our STD clinic between 2008 and 2010 was retrieved. Aetiological diagnosis of individual patients as practiced in our clinic were fit into any of the following syndromic diagnoses-vaginal/cervical discharge, non-herpetic genital ulcer, herpetic ulcer, lower abdominal pain, urethral discharge, ano- rectal discharge, inguinal bubo, scrotal swelling, genital warts, and other STIs that could not be characterised into above syndromes. The syndromic approach as suggested by National AIDS Control Organization of India was followed. Trend of syndromic diagnosis of STIs was determined.

Results In 2008, majority (51.1\%) of the new patients registered to the clinic $(36,26.66 \%$ herpetic genital ulcer, 33, 24.44\% genital warts) had one or the other viral STI. In 2009, 43.68\% patients had viral STIs (21.35\% herpetic GUD, 22.33\% genital warts). In 2010, there was a significant increase in the incidence of viral STIs; $72.6 \%$ patients (36.94\% herpetic GUD and $35.66 \%$ genital warts) had one or the other viral syndromic diagnosis.

Conclusion In concurrence to observations worldwide, proportion of viral STIs have increased significantly recently due to widespread 
use of broad spectrum antibiotics. One more reason could be recurrent or persistent nature of these viral STIs. In our setting, incidences of herpetic GUD and genital warts were almost similar.

\section{Epidemiology poster session 1: STI trends: Trichomonas vaginalis \\ P1-S1.26 PREVALENCE OF TRICHOMONAS VAGINALIS INFECTION DURING THE FIRST TRIMESTER OF PREGNANCY IN PREGNANT WOMEN ON MATERNAL AND PERINATAL INSTITUTE}

doi:10.1136/sextrans-2011-050108.26

L Cortez. Instituto Nacional Materno Perinatal, San Juan De Miraflores, Lima, Peru

Summary Objective To determine the prevalence of Trichomonas vaginalis infection during the first trimester of pregnancy in pregnant women in a Maternity Institute.

Methods We contacted and recruited a total of 105 women with up to 3 months gestation who attended the outpatient. All of them filled out a consent form and were collected a sample of vaginal secretion, which was analysed for direct observation and inoculated into the culture medium InPouch TV (Biomed Diagnostics, White City, Oregon, USA) and incubated for up $72 \mathrm{~h}$ at $37^{\circ} \mathrm{C}$. The samples were observed under the microscope for the presence of mobile parasites. Besides demographic data were collected.

Results The prevalence of infection with $T$ vaginalis was $5.71 \%$ and $3.8 \%$ by culture and direct observation, respectively. The growing use of laboratory performance improvement in the report of the prevalence of $T$ vaginalis. Close supervision of this parasite during pregnancy using more sensitive laboratory methods see Abstract P1-S1.26 Figure 1.

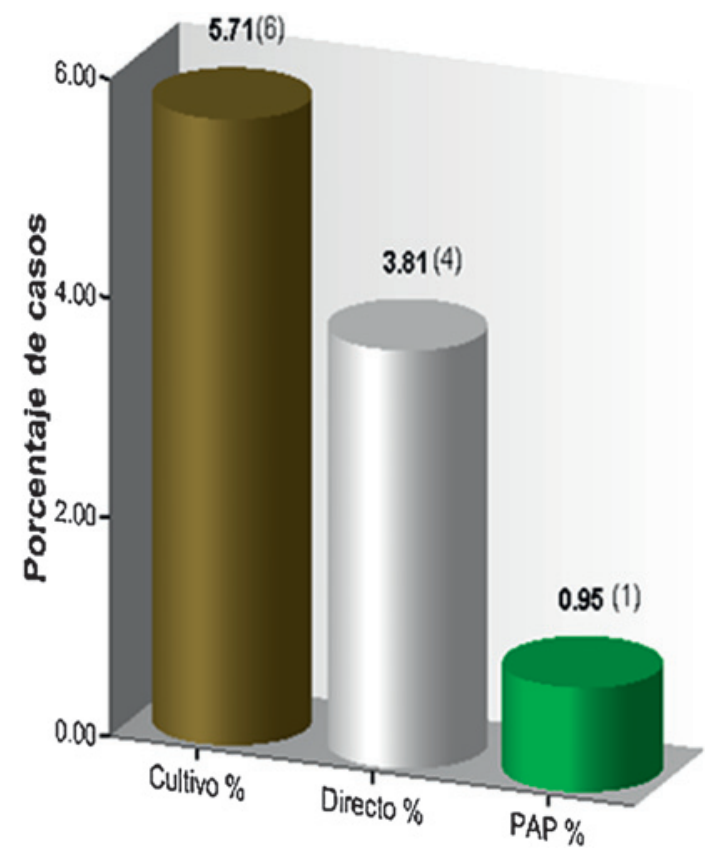

Abstract P1-S1.26 Figure 1 "Prevalence of Trichomonas vaginalis infection during the first trimester of pregnancy in pregnant women". Instituto Especializado Materno Perinatal. Lima, Mayo, Julio 2004.
Epidemiology poster session 1: STI trends: Vaginosis

\section{P1-S1.27 INTRAVAGINAL PRACTICES, LUBRICATION, AND BACTERIAL VAGINOSIS AMONG WOMEN IN LOS ANGELES}

doi:10.1136/sextrans-2011-050108.27

${ }^{1} \mathrm{~J}$ Brown, ${ }^{1} \mathrm{~K}$ Hess, ${ }^{2} \mathrm{~S}$ Brown, ${ }^{2} \mathrm{C}$ Murphy, ${ }^{2} \mathrm{~A}$ Waldman, ${ }^{2} \mathrm{M}$ Hezareh. ${ }^{1}$ University of California, Los Angeles, Los Angeles, USA; ${ }^{2}$ AIDS Research Alliance, USA

Background Bacterial vaginosis (BV) has been associated with HIV acquisition and transmission. Intravaginal practices may affect women's vulnerability to BV and HIV/STIs, and may influence results of trials of microbicides for HIV prevention through effects on the vaginal environment and on adherence to investigational microbicidal products. We measured the prevalence and frequency of intravaginal hygiene and lubricant use and their association with BV among both HIV-positive and HIV-negative women.

Methods Between October 2008 and June 2009, we recruited women aged 18 years and older in Los Angeles. At the enrolment visit and 12 months later, participants underwent a self-administered, web-based questionnaire covering demographics, sexual behaviours, intravaginal hygiene, and lubricant use. HIV was diagnosed by rapid ELISA, with confirmation by Western blot. BV was diagnosed by Nugent criteria from a self-collected vaginal swab. Here we report data collected at enrolment.

Results We enrolled 141 women (34\% identified as White, 40\% Black, 26\% Hispanic; the median age was 33 years (IOR=25-44)). Overall, $56 \%$ of women reported intravaginal cleaning and $45 \%$ reported douching over the past month. Overall, nearly threequarters $(73.8 \%)$ reported intravaginal insertion of a lubricating product over the past month; commercial lubricant (42.6\%), lotion $(12.1 \%)$, petroleum jelly $(10.6 \%)$, and oil $(7.8 \%)$ were the most commonly reported lubricants. The prevalence of BV was $21 \%$ and HIV was $27 \%$. In multivariable analysis, intravaginal insertion of oil, lotion, or petroleum jelly ( $\mathrm{aOR}=3.4$ (95\% $\mathrm{CI}=1.2$ to 9.7$)$ ) was associated with BV controlling for douching, age, race, HIV status, and multiple sexual partners.

Conclusion Use of intravaginal lubricating products not specifically designed for sexual intercourse is common among women. The use of such lubricants and their association with BV is of particular concern and warrants further study; not only in light of the direct association with BV, but also because of the link between BV and HIV infection.

\section{P1-S1.28 THE PREVALENCE AND INCIDENCE OF BACTERIAL VAGINOSIS IN A COHORT OF YOUNG AUSTRALIAN WOMEN}

doi:10.1136/sextrans-2011-050108.28

${ }^{1} \mathrm{~J}$ Walker, ${ }^{1} \mathrm{~J}$ Hocking, ${ }^{1} \mathrm{C}$ Fairley, ${ }^{2} \mathrm{~S}$ Tabrizi, ${ }^{3} \mathrm{M}$ Chen, ${ }^{4} \mathrm{~F}$ Bowden, ${ }^{1} \mathrm{~J}$ Gunn ${ }^{5} \mathrm{~B}$ Donovan, ${ }^{5} \mathrm{~J}$ Kaldor, ${ }^{3} \mathrm{C}$ Bradshaw. ${ }^{1}$ University of Melbourne, Melbourne, Australia, ${ }^{2}$ The Royal Women's Hospital, Parkville, Australia; ${ }^{3}$ Melbourne Sexual Health Centre, Carlton, Australia; ${ }^{4}$ Australian National University, Canberra, Australia; ${ }^{5}$ National Centre in HIV Epidemiology and Clinical Research, Coogee, Australia

Background Bacterial vaginosis (BV) is a common condition of unknown aetiology that can be associated with late miscarriage, preterm delivery, PID and increased susceptibility to HIV \& other STIs. In Australia, treatment is restricted to women who are symptomatic or pregnant or undergoing gynaecological treatment, however, recurrent infection is common following treatment. Currently, there are no community based BV prevalence or incidence data for Australia. 